\title{
Standards of documentation at laparoscopic entry
}

\author{
M. Raheem • Yousri Afifi
}

Received: 24 January 2008 / Accepted: 28 March 2008 / Published online: 20 May 2008

(C) Springer-Verlag 2008

\begin{abstract}
The potential for laparoscopic entry to cause serious injury is real. However, when the written records of laparoscopic entry was audited against the Middlesbrough consensus guidelines in a large teaching hospital, there were wide variations in the documentation of safety techniques. A standard proforma was created to facilitate the documentation of techniques.
\end{abstract}

Keywords Laparoscopic entry · Documentation

\section{Introduction}

Laparoscopic-specific complications are real and are associated with the creation of a pneumoperitoneum, as sharp instruments are inserted blindly into the abdomen. The potential complications ranged from minor, such as superficial bruising and subcutaneous emphysema, to major gastrointestinal and vascular injury, including gas embolism and unintended emergency laparotomy.

Large-scale reviews reported gastrointestinal injuries in the order of 0.4 to 0.7 and vascular injuries of 0.4 cases per 1,000 procedures [1], although single hospital

\footnotetext{
M. Raheem $(\bowtie) \cdot$ Y. Afifi

Birmingham Women's Hospital,

Birmingham B15 2TG, UK

e-mail: shamsuideen@yahoo.com

Y. Afifi

e-mail: yousri.afifi@bwhct.nhs.uk
}

data suggests a higher risk of bowel injury of around 3/ 1,000 procedures [2].

Since no needle-trocars system can guarantee the avoidance of injury during laparoscopic entry, adhering to consensus safeguard measures should be employed and documented when performing laparoscopic surgery. Claims related to gynaecology laparoscopic injury with the perforation of viscera or vascular injury was the most frequent (24\%) adverse factor in a Medical Defence Union's review of 732 cases over seven years [3]. Similar data concerning medical liability were reported, with laparoscopic entryrelated complications comprising a fifth of all laparoscopyrelated claims [4].

Delayed recognition of laparoscopic injury is widely reported in the literature and is a major factor in the assessment of liability. Vilos [5] reported on delayed recognition in $45 \%$ (40) of cases of laparoscopic bowel injuries, associated with $67 \%$ of the litigation outcomes unfavourable to physicians.

Ultimately, the initial documentation will reflect good medical practice [6], confirm the application of safe techniques and favourably support the laparoscopist in the event of litigation.

The main consensus document currently available for guidance at laparoscopic surgery is the Middlesbrough Consensus guidelines [7]; the Royal College of Obstetricians and Gynaecologists (RCOG) is due to publish its guidance shortly. However, a survey of gynaecologists published 6 years after the Consensus guidelines were published reported that $74 \%$ of respondents were unaware of this document [8] and others showed poor compliance with its recommendations [2]. 
This audit examined our documentation with the compliance of the Middlesbrough Consensus guidelines of laparoscopy entry.

\section{Methods}

The documentation as specified in the clinical notes of all laparoscopic-related gynaecological surgery performed in May 2007 was reviewed in a large teaching hospital. The cases were obtained from the computerised operating theatre register. The local clinical governance procedures were followed to obtain case notes. Data were collected and evaluated using Microsoft Excel.

\section{Results}

\section{Demographics}

The findings of 68 patients are recorded in this audit. Three notes could not be located and four were coded incorrectly and therefore, were, excluded. The body mass index (BMI; $\mathrm{Kg} / \mathrm{m}^{2}$ ) ranged from 18 to 40 , with a mean of 25.6. The BMI was not recorded in $15 \%$ of cases (ten women; three elective and seven emergencies). Fourteen (21\%) women had previous laparotomy, consisting of eight caesarean sections and six appendicectomies. No patients had midline laparotomy.

Eighty-seven percent $(87 \%, 59)$ electives and 13\% (9) emergency procedures were performed, consisting of $30 \%$ (20) diagnostic and 70\% (48) operative procedures. Overall, consultants performed $53 \%$ (36) of all procedures, while the registrars and senior house officers (SHOs) were the primary surgeon for $41 \%$ (28) and 5\% (4) of cases, respectively.

\section{Technique}

Catheterisation was recorded prior to Veress insertion in $51 \%$ (35) of the patients (Table 1). The Veress needle was employed in $74 \%$ (50) of the cases, inserted about the umbilicus in 43 cases (intra- or subumbilical was unclear in some documentation) and its insertion site was not recorded in $37 \%$ (25) of cases. Hasson's technique was employed in one and direct primary trocar insertion in two cases. In 7\% (5) of cases, the number of attempts at Veress insertion was documented.

The Veress insertion was tested with saline in 49\% (33) of cases and no test was stated in $41 \%$ (28) of cases. An
Table 1 Summary of audit findings

\begin{tabular}{ll}
\hline Technique assessed & No. documented, $n(\%)$ \\
\hline Patient catheterised & $35(51)$ \\
Veress insertion & $50(74)$ \\
Used & $43(63)$ \\
Intraumbilical site & $5(7)$ \\
Number of attempts for correct placement & $33(49)$ \\
Saline test & $4(6)$ \\
Pressure flow $<8$ mmHg & $1(1)$ \\
Both saline and pressure & $52(76)$ \\
Insufflation pressure & $25(36)$ \\
Insufflation volume & \\
Secondary ports under vision & $26(38)$ \\
Entry & $27(40)$ \\
Exit & $29(43)$ \\
Record of port size &
\end{tabular}

initial pressure of less than $8 \mathrm{mmHg}$ was recorded as a test of intraperitoneal placement of the Veress needle in four cases and both saline and low initial insufflation pressure $(<8 \mathrm{mmHg})$ was recorded in one case.

The insufflation pressure was documented in $76 \%$ (52) of cases and the volume in 36\% (25) of cases. In 38\% (26) of cases, documentation was made regarding the secondary ports insertion under video laparoscopy and removal in $40 \%$ (27) of cases under vision.

Port sizes were recorded in $43 \%$ (29) of cases and, in $10 \%$ (7) of cases, a port size of $10 \mathrm{~mm}$ or greater (except umbilical) of the sheath was not closed; six suprapubic and one in the right iliac fossae ports.

The only complication recorded during the audit period was an accidental posterior colpotomy incision during an operative laparoscopy (resection of endometriosis).

\section{Discussion}

This audit has found considerable heterogeneity in the quality of documentation for laparoscopy, from scant to complete documentation, in this large teaching hospital. It is prudent to note that the laparoscopic surgeons at this institution hold accreditation for training in laparoscopic surgery. Laparoscopic entry has the potential for major abdominal visceral and vascular injuries, and the operation notes should reflect that safety guidelines regarding entry were adopted.

The bladder catheterisation preceding the Veress needle insertion, an elementary safety step prior to siting instruments into the peritoneal cavity, was not recorded in $49 \%$ (33) of cases. It was unclear from the notes that a Veress 
needle was actually used for insufflations in $22 \%$ (15) of cases and the insertion site was unclear in $37 \%$ (25) of cases. Of the $62 \%$ (43) of cases with about umbilical insertion, this includes both sub- and intraumbilical sites. The recommendation is to use the base of the umbilicus, as it is the thinnest and least vascular part of the anterior abdominal wall [9].

Hasson's and direct insertion of primary trocars were not commonly used and the Veress preference was not altered by the presence of abdominal scars. However, none of the women had midline abdominal scars. The consensus guidance suggested selecting an alternate site for Veress insertion in the presence of midline scars, as it reduces the incidence of type 2 visceral injury [7].

The merit of testing the Veress insertion and the best methods to employ is debated. The guidelines do recommend some form of testing and Palmer's tests, as well as the gas flow pressure rates, were reported. This audit found that, in $41 \%$ (28) of cases, no safety test for the Veress insertion was documented and the initial gas flow pressure rate was not popular. Large-scale studies have shown that an initial insufflations pressure of less than $8 \mathrm{mmHg}$ was consistent with correct intraabdominal placement of the Veress needle, regardless of the patient's body habitus [10]. Only in five cases was the number of attempts at Veress insertion documented, ranging from one to four attempts.

The insufflation pressure prior to the primary trocar insertion was not documented in $24 \%$ (16) of cases and the volume was not recorded in $60 \%$ (41) of cases. The guidelines recommend insufflation pressures of $25 \mathrm{mmHg}$, as this increase the "gas bubble," as well as creating a splinting effect on the abdominal wall [7]. Recording the volume is a form of self-checking, as studies have documented that a volume in excess of $5 \mathrm{~L}$ is necessary to achieve the required $25-\mathrm{mmHg}$ pressure. This safety precaution has been found to reduce the risk of type 1 visceral injury.

Secondary ports should be inserted and removed under direct vision and this was not stated in the documentation in $62 \%$ (42) of cases of entry and in $60 \%$ (41) of cases of removing the ports. This recommendation facilitates avoiding type one visceral injury.

Ports $10 \mathrm{~mm}$ or greater away from the umbilicus should be closed, as this reduces the risk of herniation of abdominal viscera. In $10 \%$ of cases, the port was not closed and in a further $43 \%$ of cases, the port sizes were not stated.

General observations include the common use of nonstandard abbreviations, e.g. c/d/c and udv. The notes made no mention of the patients' positioning, as the guidelines recommend the patient lying supine at Veress insertion and primary trocar entries [7]. The only complication recorded was the unintended posterior colpotomy incision during an operative laparoscopy during a difficult case of resection for endometriosis.

\section{Summary of main findings}

There were wide variations in the documentation of the recommended safety procedures for laparoscopic entry, as outlined in the Middlesbrough Consensus guidelines.

The Veress needle inserted about the umbilicus was the preferred site for pneumoperitoneum and the saline test was preferred over initial low insufflation pressures as a test for intraperitoneal placement.

Senior grade clinicians were the primary surgeons for laparoscopic procedures, with restricted opportunities for juniors.

The use of non-standard abbreviations was common and we suspect that this was done in order to facilitate speedier documentation.

Some women with port sizes $10 \mathrm{~mm}$ or greater did not have sheath closure.

\section{Recommendations}

The main recommendation from this audit was to introduce a standard proforma to systematically record that the safety recommendations of laparoscopy were applied (Appendix). This teaching hospital has a significant advantage, as the consultants with interest in minimal access surgery are also accredited trainers for laparoscopic surgery. This simple measure can be incorporated onto the current operation notes and should improve documentation with recording the practice of safety guidelines.

\section{Appendix 1: suggested proforma to be attached on the reverse of current surgical records}

\begin{tabular}{ll}
\hline $\begin{array}{l}\text { Patients position on table } \\
\text { Catheterised }\end{array}$ & yes/no \\
Veress needle & \\
Intraumbilical/subumbilical /others, specify & \\
Number of attempts of Veress insertion & \\
Double click technique & yes/no \\
Insertion angle & \\
Tested & \\
Saline & \\
Initial pressure & \\
Both &
\end{tabular}


Insufflation pressure

Insufflation volume

Technique for primary trocar

Direct entry

Hasson's entry

Secondary ports inserted under direct yes/no vision

Ports removed under direct vision yes/no

Closure of ports (sizes, suture, sheath closure and local anaesthetic use)

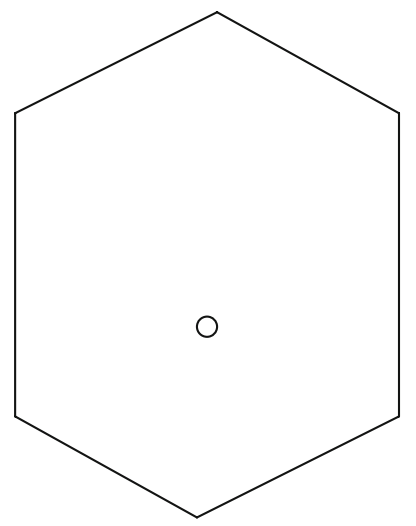

both hands on trocar one hand each on trocar/ abdomen

\section{References} entry: a literature review and analysis of techniques and complications of primary port entry. Aust N Z J Obstet Gynaecol 42(3):246-254

2. Jones KD, Fan A, Sutton C (2002) Safe entry during laparoscopy: a prospective audit in a district general hospital. Gynaecol Endosc $11(2-3): 85-89$

3. Goodwin H (1998) Minimal access surgery. J MDU 14(1):12-14

4. Wind J, Cremers JE, van Berge Henegouwen MI, Gouma DJ, Jansen FW, Bemelman WA (2007) Medical liability insurance claims on entry-related complications in laparoscopy. Surg Endosc 21:2094-2099

5. Vilos GA (2002) Laparoscopic bowel injuries: forty litigated gynaecological cases in Canada. J Obstet Gynaecol Can 24(3):224-230

6. Good Medical Practice (2006) Available online at: http://www. gmc-uk.org/guidance/good_medical_practice/index.asp

7. A consensus document concerning laparoscopic entry techniques: Middlesbrough, March 19-20 1999 (1999) Gynaecol Endosc 8 (6):403-406

8. Lalchandani S, Phillips K (2005) Laparoscopic entry techniquea survey of practices of consultant gynaecologists. Gynecol Surg 2 (4):245-249

9. Roy GM, Bazzurini L, Solima E, Luciano AA (2001) Safe technique for laparoscopic entry into the abdominal cavity. J Am Assoc Gynecol Laparosc 8(4):519-528

10. Vilos GA, Vilos AG (2003) Safe laparoscopic entry guided by Veress needle CO2 insufflation pressure. J Am Assoc Gynecol Laparosc 10(3):415-420 